\title{
Study on the Effects of Sorbitol on Water Holding Capacity of Meat by NMR
}

\author{
Xiaolei YAN ${ }^{\mathrm{a}, 1}$, Bin DU ${ }^{\mathrm{a}}$, Juan $\mathrm{LU}^{\mathrm{a}}$ and Wenkun $\mathrm{ZHANG}^{\mathrm{b}}$ \\ a Weifang Vocational College of Engineering, Qingzhou City, Shandong Province, \\ China \\ ${ }^{\mathrm{b}}$ Weifang Noted Pharmaceutical Co., Ltd., Qingzhou, Shandong, China
}

\begin{abstract}
The distribution and changes of constitutional water, immobilized water and free water and the effects of the sorbitol on three kinds of water during the curing of fresh meat were studied in this paper. The low-field Nuclear Magnetic Resonance were used to measure water and its distribution in meat. The test parameters are as follows: magnetic field strength of the NMR spectrometer is 0.5 Tesla, corresponding resonance frequency for protons is $22 \mathrm{MHz}$, sampling points is 160128 , repeated scanning number is 16 , repetition time is $1.6 \mathrm{~s}$, echo time is $1.6 \mathrm{~s}$. The results showed that: the relaxation time $\mathrm{T} 2$ can be divided into three parts: T21 (0 10ms), T22 (10 100ms), T23 (100 1000ms). And the area of T21, T22, T23 represents the contents of constitutional water, immobilized water and free water. The addition of sorbitol can keep water during curing. The sorbitol can reduce the activity of the immobilized water and free water during curing of the fresh meat.
\end{abstract}

Keywords. Water holding capacity, low-field Nuclear Magnetic Resonance, NMR relaxation method

\section{Introduction}

The main components and the amounts in animal muscles are: water $65 \%-80 \%$, protein $16 \%-22 \%$, carbohydrate $1 \%-2 \%$, fat $1 \%-13 \%$, other soluble material $1 \%$. Water is a key component which strongly affects the chemical changes in food system, for example, protein denaturation [1] and enzyme activity [2] and it is also a determinant of the rheological behavior and is widely involved in chemical and physical changes [3]. Water is necessary for the growth of microorganisms and enzymatic activities. So water can affect the stability and shelf life of food system. Therefore, water activity (Aw) has been used to indicate how the microorganisms survive and grow for many years. However, food is an inhomogeneous system and water is located in compartments within the structure. Compared with the overall water activity of the production system, a compartment filled with water may have a different local Aw [4]. So it is very important to create a local environment which can increase the growth and survival of the microorganisms in food. But the research on moisture behavior in food system is not easy. The advantage of the NMR relaxation method is that it can provide rapid, potentially non-destructive and non-invasive information together with the

${ }^{1}$ Corresponding Author, Xiaolei YAN, Weifang Vocational College of Engineering, Qingzhou City, Shandong Province, China; Email: xiaoleikx1@163.com. 
information of division and mobility of moisture in meat $[5,6]$. There are many reports of the use of Nuclear magnetic resonance to measure water and its distribution in meat, especially in pork muscle [7]. Now, more and more food scientists are interested in the new technology's application in food science.

The spatial resolution provided by magnetic resonance imaging (MRI) allows $1 \mathrm{H}-$ MRI to characterize tissue morphology. Without any demand for preliminary sample preparation, the MRI technology can obtain two-dimensional or three-dimensional internal images non-invasively and non-destructively [8]. The useful of MRI allows the spatial resolution of water content. AND the NMR parameters, such as relaxation time (for example T1 and T2) together with the diffusion coefficient (D), define the state of water interacting with other molecules. Due to changes in water-macromolecule interactions and changes in organizational structure, the parameters are very sensitive to local changes in water mobility.

\section{Material and Methods}

\subsection{Curing of Fresh Meat}

First, cut the meat into $1 \mathrm{x} 1 \mathrm{~cm}$ cubes, after this, put them into a handy container, then according to the following solutions to cure them by injection: (1) $20 \%$ water (compared to the weight of the fresh meat), 3\% salt; (2) $20 \%$ water, $3 \%$ salt, $1 \%$ sorbitol; (3) $20 \%$ water, $3 \%$ salt, $5 \%$ sorbitol; (4) $20 \%$ water, $3 \%$ salt, $8 \%$ sorbitol; (5) $20 \%$ water, $3 \%$ salt, $10 \%$ sorbitol. Cure the meat for $36 \mathrm{~h}$ in the $4{ }^{\circ} \mathrm{C}$ fridges.

\subsection{NMR Experiments}

We use NMR and MRI measurements to do the experiment. And the RINMR/MRI spectroscope equipped with gradient amplifiers and temperature control devices and a large bore NMR/MRI system. The samples were positioned in the homogeneous region of the probe head. The magnetic field strength of the NMR spectrometer is 0.5Tesla, and the corresponding resonance frequency for protons is $22 \mathrm{MHz}$.

Other test parameters are as follows: Sampling points is 160128; repeated scanning number is 16 ; repetition time is $1.6 \mathrm{~s}$; echo time is $1.6 \mathrm{~s}$. The analysis of relaxation date was performed using the Win-MRIXP Programme (East China Normal University, Shanghai, China).

NMR Experiments of the Cured Meat. After the meat were cured for 36 hours, a small piece of meat was removed and weighted. We should record the weight and wrap the meat with raw materials. Raw materials were used to wrap the meat due to they have no proton signals. Then the samples were put into the NMR Analyzer, and the T2 relaxation of the sample were performed by the Win-MRIXP Programme. And the MRI measurement of the cured meat were also made together.

\subsection{Data Analysis}

We use SPSS software package, release 11.5 standard version to analyse the data. 


\section{Results and Discussion}

\subsection{The NMR Figure of Cured Meat}

NMR figure of the cured materials is shown in figure 1. What we find experimentally is that there are 3 or 4 peaks in samples [9]. So we can divide the relaxation time T2 into three parts: T21 $(0 \sim 10 \mathrm{~ms})$, T22 $(10 \sim 100 \mathrm{~ms})$, T23 $(100 \sim 1000 \mathrm{~ms})$. And this is in line with the results with of R. Miklos; H. Mora-Gallego [10]. The three components showed in the figure were designated as extracellular wate r(T23), myofibril and reticulated water (T22), and water which in interact with macromolecules (T21). The longest T2 was considered to correspond to expelled water. And the major part of the water is believed to be held by myofibrils in food system [11]. In fact, water is bound in different degrees. The T2 represents the ability of the water mobility, and the more closely the water were bound, the shorter the relaxation time (T2).

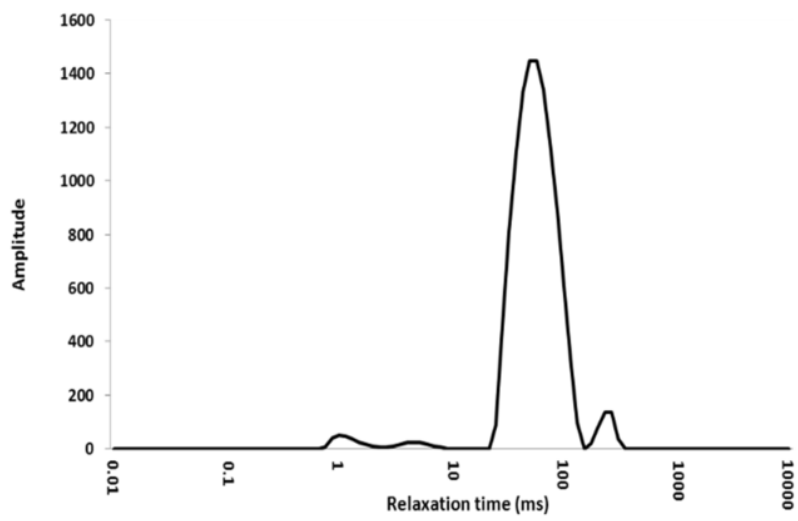

Figure 1. The NMR figure of raw material.

\subsection{Effect of the Sorbitol on the Cured Meat}

We take a certain amount of fresh meat, and cure the meat by injection according to the following formula: $20 \%$ water (compared to the weight of meat) (1) $20 \%$ water, $3 \%$ salt; (2) $20 \%$ water, $3 \%$ salt, $1 \%$ sorbitol; (3) $20 \%$ water, $3 \%$ salt, $5 \%$ sorbitol; (4) $20 \%$ water, $3 \%$ salt, $8 \%$ sorbitol; (5) $20 \%$ water, 3\% salt, $10 \%$ sorbitol. After 36 hours, the NMR and MRI experiment were done according the 2.2 parameters.

From table 1, we found that the relaxation area of T21, T22 did not change a lot. But the addition of sorbitol can increase the relaxation area of T23. We know that T21, T22, T23 stand for the water in interaction with macromolecules, water in myofibrils and reticulum and the extracellular water respectively. For we cured the meat by injection, so we can think that the sorbitol can help meat keep and maintain water from outside. From table 1, we found that the total relaxation area is also enlarged. It meant that the total water in meat were increased by the addition of the sorbitol.

The NMR relaxation studies can evaluate the meat characteristics, but the disadvantage is that it cannot determine the location in the muscle. But he MRI method provides a picture of relaxation time in tissues on the supramolecular scale. Diffusion MRI is a mature tool [12] for non-invasive study of muscle structure. 
Table 1. The effect of the sorbitol on the relative area and time of the cured meat.

\begin{tabular}{|c|c|c|c|c|c|c|c|}
\hline \multirow[b]{2}{*}{$\begin{array}{l}\text { Sorbitol } \\
\text { content }\end{array}$} & \multicolumn{2}{|c|}{$\begin{array}{l}\text { RelativeRelaxation time } \\
\text { area }(\mathrm{ms})\end{array}$} & \multicolumn{2}{|c|}{$\begin{array}{l}\text { RelativeRelaxation time } \\
\text { area }(\mathrm{ms})\end{array}$} & \multicolumn{2}{|c|}{$\begin{array}{l}\text { Relative Relaxation time } \\
\text { area }(\mathrm{ms})\end{array}$} & \multirow{2}{*}{$\begin{array}{l}\text { Relative } \\
\text { area }\end{array}$} \\
\hline & $\mathbf{T} 21$ & & $\mathbf{T} 22$ & & $\mathbf{T} 23$ & & \\
\hline $0 \%$ & 455 & 4.037 & 14591 & 57.224 & 50 & 351.119 & 15096 \\
\hline $1 \%$ & 494 & 4.641 & 14475 & 49.770 & 78 & 351.119 & 15047 \\
\hline $5 \%$ & 447 & 3.511 & 14452 & 49.770 & 318 & 351.119 & 15217 \\
\hline $8 \%$ & 442 & 4.037 & 14422 & 57.224 & 421 & 351.119 & 15285 \\
\hline $10 \%$ & 460 & 3.054 & 14846 & 49.770 & 549 & 305.119 & 15855 \\
\hline
\end{tabular}

Note: the relative area means the relaxation per unit mass; different letters within the column indicate statistical differences $(P<0.05)$; all index were measured three times. The results are: the means \pm standard error.

There is a proportionality between the signal strength and proton density. Normally, the stronger the signal of the area, the brighter the image is. And the proton density is bigger also. Of course, it is the same in reverse [13]. Compared figures $2 \mathrm{a}, 2 \mathrm{~b}$ and $2 \mathrm{c}$, we can see that the image becomes brighter gradually. So, the water content of the three samples is: figure $2 \mathrm{c}$ (the meat cured with $10 \%$ sorbitol) $>$ figure $2 \mathrm{~b}$ (the meat cured with $5 \%$ sorbitol) $>$ figure 2 a (the meat cured with $0 \%$ sorbitol). And we can consider that the sorbitol can keep the water. For the only way to deal with the meat is curing them by injection, the increased water is from outside. And certainly, we can think that the sorbitol can help to keep the water from outside. And this result is consistent to that form table 1 . The white lines in figure $2 \mathrm{a}$ are muscle fibers in meat. And in figure $2 b$, there is a brighter area on the edge of the image. I think there are two reasons: when the meat was taking out, the water on the surface was not dried completely. The other is that the meat was cured at $4{ }^{\circ} \mathrm{C}$, when put to the experiment environment $28^{\circ} \mathrm{C}$, part of the water exuded. For 5\% sorbitol can't keep the water tightly enough.

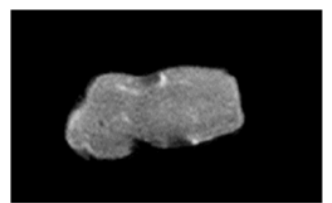

(a)

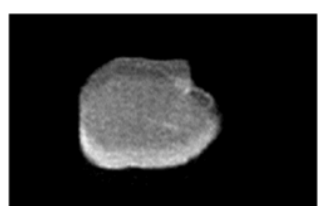

(b)

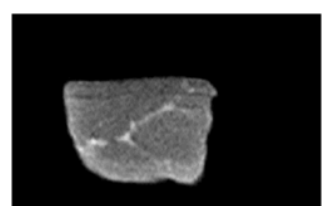

(c)

Figure 2. The magnetic resonance image of the cured meet: (a) cured with $0 \%$ sorbitol; (b) cured with $5 \%$ sorbitol; (c) cured with $10 \%$ sorbitol.

\section{Conclusion}

The present study showed that the relaxation time T2 can be divided into three parts: T21 (0 10ms), T22 (10 100ms), T23 (100 1000ms). And the area of T21, T22, T23 represents the contents of constitutional water, immobilized water and free water. And changes in the distribution and mobility of water caused by the sorbitol in cured meat were detectable by NMR T2 relaxation and the magnetic resonance image, where the sorbitol caused a shift towards faster relaxation times and a larger relative area of T23. The sorbitol can reduce the activity of the immobilized water and free water during the curing of fresh meat. 


\section{References}

[1] Xia T L and Liu D Y 2011 Application of low-field nuclear magnetic resonance in determining water contents and other related quality characteristics of meat and meat products Food Sci. (32) 253-256.

[2] Cui H B and Xue C H 2013 Development of shelf-stable, ready-to-eat shrimps using water activity lowering agent by response surface methodology Journal of Food Science and Technology (6) 11371143.

[3] Zhang J S, Lin X Y, Ruan R S and Qi J N 2006 Mobility of water in binary system of bread and cheese as studied by magnetic resonance imaging Food Science (27) 132-138.

[4] Moller S M, A Gunvig and Bertram H C 2010 Effect of starter culture and fermentation temperature on water mobility and distribution in fermented sausages and correlation to microbial safety studied by nuclear magnetic resonance relaxometry Meat Science (86) 462-467.

[5] Hazlewood C F, Chang D C and Nichols B L 1974 Nuclear magnetic resonance transverse relaxation times of water protons in skeletal muscle Biophysical Journal (14) 583-606.

[6] Lin W Y, Lin J J and Lin X Y 2013 Effect of salt on surimi processing explored by nuclear magnetic resonance Food Sci. (34) 105-109.

[7] Brondum J, Munck L, Henckel P, et al. 2000 Prediction of water-holding capacity and composition of porcine meat by comparative spectroscopy Meat Science (55) 177-185.

[8] Renou J P, Foucat L and Bonny J M 2003 Magnetic resonance imaging studies of water interactions in meat Food Chemistry (82) 35-39.

[9] Bertram H C, Karlsson A H and Andersen H J 2003 The significance of cooling rate on water dynamics in porcine muscle from heterozygote carriers and non-carriers of the halothane gene-a low-field NMR relaxation study Meat Science (65) 1281-1291.

[10] Miklos R, Mora-Gallego H, et al. 2014 Influence of lipid type on water and fat mobility in fermented sausages studied by low-field Meat Science (96) 617-622.

[11] Bertram H C, Andersen H J and Karlsson A H 2001 Comparative study of low-field NMR relaxation measurements and two traditional methods in the determination of water holding capacity of pork Meat Science (57) 125-132.

[12] Le Bihan D, Mangin J F and Poupon C 2001 Diffusion tensor imaging: Concepts and applications Journal of Magnetic Resonance Imaging (13) 534-546.

[13] Lin X Y, He C Y, Ruan R S, et al, 2005, Studies of water mobility in frozen steamed bread under microwave reheating using magnetic resonance Imaging Food Science (26) 82-86. (in Chinese) 